\title{
FORMACIÓN DE INNOVADORES EN EL DESARROLLO DE APLICATIVOS MÓVILES. LA EXPERIENCIA APPS.CO EN SANTANDER
}

\author{
Adriana Rocío Lizcano ${ }^{1}$, Armando Arévalo², Sergio Andrés Zabala ${ }^{3}$ \\ ${ }^{1}$ Docente investigadora, Grupo de Investigación en Nuevas Tecnologías aplicadas a la Educación-GIDSAW \\ Correo electrónico: alizcano@udi.edu.co \\ 2 Director de la Dirección de Investigaciones \\ ${ }^{3}$ Docente investigador, Grupo de Investigación en Robótica, Control y Procesamiento de Señales \\ Universitaria de Investigación y Desarrollo, Bucaramanga, Colombia
}

Recibido: 15 de agosto del 2013. Aprobado: 5 de octubre del 2013.

Cómo citar este artículo: A. R. Lizcano, A. Arévalo y S. A. Zabala, "Formación de innovadores en el desarrollo de aplicativos móviles. La experiencia Apps.co en Santander". Ingeniería Solidaria, Vol. 9, No. 16, pp. 49-55, Dic., 2013.

Resumen. Apps.co es una iniciativa del Ministerio de Tecnologías de la Información y las Comunicaciones (Mintic) y su plan Vive Digital para promover y potenciar la creación de negocios a partir del uso de las Tic, enfocados en el desarrollo de aplicaciones móviles, software y contenidos. La meta gubernamental es la generación de mínimo 400 soluciones en mercados internacionales. El programa Apps.co está compuesto por cuatro fases: Bootcamps, Ideación, Prototipado y Validación, Aceleración y Consolidación. El Gobierno Nacional recurrió a aliados estratégicos como ejecutores del proceso. En Ideación, Prototipado y Validación, Santander cuenta con: 1. Apps.co unAB y 2. SantanderApps (Unión Temporal entre UPB, UDI y CETICs). Cada uno de ellos ha coordinado 20 emprendimientos por iteración, asesorándolos en aspectos técnicos y empresariales, durante ocho semanas de acompañamiento para consolidar la idea, el modelo de negocio y generar un prototipo de la aplicación. Este artículo sintetiza los aspectos metodológicos y técnicos que se han implementado en la fase de "Ideación, prototipado y validación" por la alianza SantaderApps, finalizando con una presentación descriptiva de los resultados obtenidos y las lecciones aprendidas, con el objetivo de generar una base de conocimiento que pueda ser aprovechada para generar procesos de emprendimiento tecnológico por diferentes actores del sistema productivo y educativo nacional.

Palabras clave: desarrollo de apps, emprendimiento TIC, lean launch pad, Ministerio TIC.

Creating Innovators in Mobile Application Development. The Experience of Apps.co in Santander

Abstract. Apps.co is an initiative of the Ministry of Information Technology and Communication (MinTIC) and its Vive Digital (Live Digital) plan for promoting, through the use of information and communication technology (ICT), the creation of businesses that focus on developing mobile applications, software and content. The government's goal is to generate at least 400 solutions in international markets. The Apps.co program consists of four phases: Bootcamps, Conceptualization, Prototyping and Validation, Acceleration and Consolidation. The National Government involved strategic allies to execute this process. In Conceptualization, Prototyping and Validation, Santander has: 1. Apps.co UNAB and 2. SantanderApps (a joint venture between UPB, UDI and CETICS). Each of these has coordinated 20 startups per iteration, providing technical and business consulting over an eight-month support period to consolidate the idea and the business mod$\mathrm{el}$, and create a prototype of the application. This article summarizes the methodological and technical aspects of work done by the SantanderApps alliance in the "Conceptualization, Prototyping and Validation" phases. The article finishes with a description of the results and lessons learnt in order to create a knowledge base that can be used by different players in the economy and the national education system for technology entrepreneurship processes.

Keywords: application development, ICT entreprenuership, lean launch pad, Ministry of ICT.
FORMANDO INOVADORES NO DESENVOLVIMENTO DE aplicativos Móveis. A EXPERIÊNCIA APPS.co EM SANTANDER

Resumo. Apps.co é uma iniciativa do Ministério de Tecnologias da Informação e das Comunicações (MinTic) e seu plano Vive Digital para promover e potencializar a criação de negócios a partir do uso das TIC, enfocados no desenvolvimento de aplicativos móveis, software e conteúdos. A meta governamental é a geração de mínimo 400 soluções em mercados internacionais. O programa Apps.co está composto por quatro fases: Bootcamps, Concepção, Prototipagem e Validação, Aceleração e Consolidação. O Governo Nacional recorreu a aliados estratégicos como executores do processo. Em Concepção, Prototipagem e Validação, Santander conta com: 1) Apps.co UNAB e 2) SantanderApps (União Temporal entre UPB, UDI e CETICS). Cada um vem coordenando 20 empreendimentos por iteração, assessorando-os em aspectos técnicos e empresariais, durante oito semanas de acompanhamento para consolidar a ideia, o modelo de negócio e gerar um protótipo da aplicação. Este artigo sintetiza os aspectos metodológicos e técnicos que se foram implantados na fase de "concepção, prototipagem e validação" pela parceria SantaderApps, e finaliza com uma apresentação descritiva dos resultados obtidos das lições aprendidas, com o objetivo de gerar uma base de conhecimento que possa ser aproveitada para gerar processos de empreendimento tecnológico por diferentes atores do sistema produtivo e educativo nacional.

Palavras-chave: desenvolvimento de apps, empreendimento TIC, lean launch pad, Ministério TIC. 


\section{Introducción}

Es innegable el impacto de las Tecnologías de la Información y la Comunicación (TIC) en el desarrollo económico de los países. En América Latina algunos de ellos han realizado una apuesta importante por la modernización del Estado, la conectividad, la formación de capital humano y el fortalecimiento del emprendimiento tecnológico, entre ellos México, Chile y, en los últimos años, Colombia. Adicionalmente, según lo planteado en el Informe Horizon 2012 generado por New Media Consortium [1] y los aportes sobre uso de aplicaciones de Burton Lee [2] existe una alta tendencia internacional hacia el desarrollo e innovación en aplicaciones móviles en diferentes áreas, y el reto gira alrededor de consolidar clientes, generar necesidad, realizar desarrollos efectivos y conectar los diferentes entes regionales o nacionales.

En la actualidad, el gobierno colombiano se encuentra ejecutando programas como Vive Digital y Apps.co, entre otros, liderados por el Ministerio de Tecnologías de la Información y las Comunicaciones (MinTIC). Estos giran en torno a la incorporación, mejoramiento, capacitación y generación de emprendimiento en áreas propias de la gestión de las TIC como herramientas generadoras de desarrollo.

En el caso específico de Apps.co, se pretende capacitar y consolidar ideas innovadoras centradas en aplicaciones móviles, con un grupo de emprendedores apoyados por entidades aliadas del MinTIC para su formación y acercamiento a clientes potenciales, así como en el conocimiento de herramientas organizacionales y técnicas para lograr que su idea de negocio sea una realidad [3]. El programa Apps.co está compuesto por cuatro fases:

- Bootcamps: un conjunto de cursos de entrenamiento en lenguajes de programación para el desarrollo de aplicaciones en web y plataformas móviles nativas como Android, Microsoft e iOS, siguiendo metodologías de desarrollo ágil y por niveles, de acuerdo con la experticia de los interesados.

- Ideación, Prototipado y Validación: esta fase se concentra en mejorar las habilidades para identificar oportunidades de negocio. En esta fase se proporciona orientación para validar el aplicativo en el mercado, empezando por prototipos rápidos hasta llegar a un producto que permita tener los primeros clientes o usuarios.
- Aceleración: consiste en acompañar empresas TIC con mínimo un año de constitución, interesadas en escalar su negocio rápidamente, mejorando su capacidad interna para crear valor, mediante el apoyo de expertos internacionales.

- Consolidación: en esta fase se ofrece acompañamiento y asesoría en el proceso de escalamiento y consolidación de un negocio a empresa. Aquí se incorporan emprendedores con un producto o servicio validado dentro de la industria TIC, para que durante 14 semanas adquieran más clientes, ajusten su modelo de negocio, consoliden su empresa y entren en un ritmo de crecimiento sostenido.

Este proceso de formación de emprendedores se hace aún más relevante para la situación particular del departamento de Santander, donde el Plan de Desarrollo Departamental 2012-2015 [4] cita la importancia del desarrollo y fomento de la incorporación y apoyo de las herramientas TIC para la competitividad de la región, o en otras estrategias de entidades regionales como el Clúster de Empresas de Tecnologías de Información y las Comunicaciones de Santander, que consideran este tipo de estrategias como prioritarias para la región.

Lo anterior se apoya en cifras a nivel internacional que indican que las proyecciones mas realistas plantean más de 1000 millones de smartphones en el mercado mundial [5], que deben tenerse en cuenta en el desarrollo de productos y servicios, con el fin de explotar todas sus potencialidades.

Específicamente, en la fase de Ideación, Prototipado y Validación el Mintic ha seleccionado la metodología Lean LaunchPad [6] para su implementación. El Lean LaunchPad proporciona un conjunto de actividades que orientan a las startup para lograr que su modelo de negocio sea repetible y escalable, y parte de la definición de startup como una organización temporal cuyo fin principal es refinar ese modelo de negocio.

El punto de partida para la implementación del Lean LaunchPad son las ideas de negocio orientadas hacia el desarrollo de aplicaciones móviles (apps) formuladas por equipos de dos a cuatro personas, con diferentes perfiles, conocimientos y perspectivas, que se incorporan al programa Apps.co, mediante un proceso de inscripción y selección que se realiza vía web. La duración del proceso es entre siete u ocho semanas de acompañamiento realizado por un conjunto de mentores, en pro de lograr el objetivo de obtener un modelo de negocio repetible y escalable. 
Una revisión de antecedentes de la metodología Lean LaunchPad en bases de datos (EBSCO, Elsevier, entre otros), proporciona información muy reducida que no aborda los factores asociados con su implementación, mucho menos en el estudio, comprensión y adaptación de la misma al caso colombiano. En este sentido, surge la necesidad de realizar un seguimiento detallado a la implementación de dicha metodología y la generación de buenas prácticas para lograr las metas de emprendimiento y desarrollo planteadas por el programa Apps.co y el MinTic, y se originen lineamientos que puedan ser aprovechados para generar procesos de emprendimiento orientados al desarrollo tecnológico e innovación.

Para presentar dicho seguimiento, este artículo ofrece una descripción de las tendencias sobre el emprendimiento TIC, especialmente orientado hacia el desarrollo de aplicativos para dispositivos móviles, a modo de preámbulo para justificar la importancia de las iniciativas orientadas hacia esta tecnología. Posteriormente, describe el proceso de implementación que ha seguido la Unión Temporal UDI-UPB-CETICs en el programa Apps.co, para finalizar con la identificación de las lecciones aprendidas que orienten la aplicación del modelo en posteriores iteraciones o en procesos de formación de emprendedores con un núcleo de negocios orientados hacia la tecnología.

\subsection{Tendencias de emprendimiento TIC}

Es creciente el número de ejemplos asociados a proyectos y productos que buscan consolidar el desarrollo de aplicaciones orientados a los teléfonos celulares y las tabletas. La búsqueda en bases de datos especializadas proporcionan un conjunto de experiencias sobre aplicativos móviles, como es el caso del trabajo denominado Invasion of the Mobile Apps [7], en el que Anthes muestra cómo el incremento de las aplicaciones -en este caso para IPhone y Android- han modificado el mercado y sus usuarios. En este trabajo se destaca el término de startup con el objetivo de establecer y propiciar desarrollos empresariales, basados en la organización de propuestas de rápida implementación, con clientes potenciales conocidos que permiten ajustar de forma ágil el aplicativo final según los requerimientos del usuario o grupos de usuarios.

Otro trabajo asociado al desarrollo de aplicaciones móviles es denominado Best Apps [8], en el cual se revi- sa un conjunto de aplicaciones exitosas publicadas en el 2012, destacando aportes en el área aeronáutica (NASA), médica (Medical Joyworks), posicionamiento y ubicación global (Vito Tech), entre muchas otras.

Por otra parte, en el área académica y educativa, se encuentran varios trabajos descriptivos sobre aplicaciones, la experiencia de su desarrollo y, en algunos casos, el modelo utilizado para la consolidación de las mismas. Algunos ejemplos de estos trabajos se vinculan al trabajo con pantallas táctiles en la educación [9], el uso de dispositivos móviles para la enseñanza de la matemática [10], ejemplos del uso de tabletas - en este caso específicas al IPad- en el salón de clase [11] y algunos destacados trabajos y experiencias en España y Latinoamérica como la destacada por Contreras, $\mathrm{He}-$ rrera y Montoya [12]. Este último trabajo se destaca por realizar un proceso detallado de análisis y evaluación de materiales educativos con tecnología celular, recurriendo a sesiones grupales, análisis a través de encuestas y registros de observación.

Dentro del contexto de aplicaciones específicas para la consolidación de sectores diferentes al educacional, se destacan el desarrollo de aplicaciones hacia el sector de las leyes, donde se pueden encontrar al menos trece aplicaciones móviles que simplifican y automatizan muchos procesos del sector [13]; o el aporte que pueden realizar las aplicaciones móviles en la enseñanza del emprendimiento, caso de estudio de la Universidad Politécnica de Bucharest [14], en la cual se evalúa el impacto de estas herramientas en diferentes proyectos de cerca de 2.500 beneficiarios.

Por otra parte, se encuentra un conjunto de trabajos que permiten evidenciar las altas proyecciones económicas del sector de desarrollo de apps realizados durante los últimos dos años. Dentro de estos se destaca el de Jason del Rey [15], que describe las principales sedes a nivel mundial de desarrollo y emprendimiento en esta temática, y presenta cifras que proyectan los mercados de comercialización de aplicaciones logrará como un negocio de más de 40 billones de dólares en el 2014.

De lo anterior se puede concluir que existe una marcada tendencia hacia la producción de materiales para aplicativos móviles, lo que hace necesario proyectar procesos de formación para el desarrollo de los mismos y la generación de emprendimientos competitivos y sostenibles. 


\section{Metodología}

La metodología Lean LaunchPad (LLP) [6], seleccionada por el Mintic para su aplicación en el programa Apps. co del caso colombiano, es un proceso que tiene como fin estimular, apoyar y acelerar empresas, para hacer un tránsito más efectivo desde una idea hacia la realidad de la empresa minimizando el riesgo. LLP ha sido usada exitosamente por la Universidad de Stanford para apoyar iniciativas TIC, basada en el principio de desarrollo de clientes para lograr alinear las funcionalidades del producto con las necesidades y deseos reales del cliente.

LLP define tres aspectos claves: estrategia, proceso y organización, que se implementan ya sea para crear una nueva startup o para definir una nueva unidad de producto en una organización.
La estrategia se centra en plantear y probar la hipótesis del modelo de negocio, basada en un proceso orientado a la organización e implementación de dicha búsqueda del modelo de negocio a través de procesos de desarrollo de cliente y desarrollo ágil; finalmente, en cuanto a la organización, el LLP se centra en definir el equipo de desarrollo de clientes liderado por los fundadores y creadores de la idea.

Estos tres aspectos requieren de la formación de los diferentes actores del proceso en unos temas específicos y la utilización de unas estrategias pedagógicas que aseguren la asimilación y aplicación de dichos conceptos en la iniciativa particular para lograr su evolución. La figura 1 muestra la interacción de dichos aspectos.

\section{Temas de formación}

Diseño del modelo de negocio, desarrollo de producto/servicio, desarrollo de clientes, conformación del equipo de startup, finanzas empresariales, mercadeo, transición del fundador, etc.

\section{Estrategia de formación}

Clases experienciales, investigativas y centradas en el estudiante
- Plantear y probar una hipótesis del modelo de negocio

- Herramienta: Business model canvas

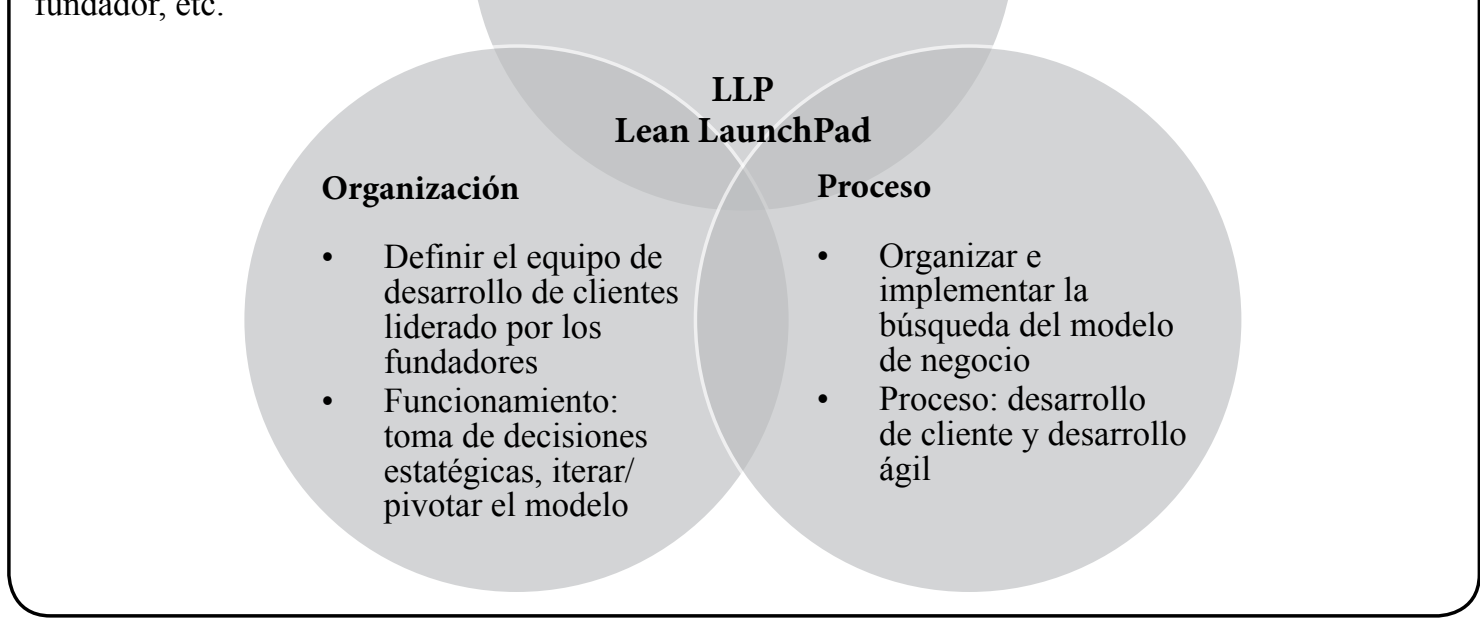

Figura 1. Componentes del Lean LaunchPad

Fuente: los autores

La herramienta fundamental que permite representar y comunicar la hipótesis y que se constituye en el punto focal de la discusión es el Business Model Canvas [16], un esquema que consta de nueve bloques que constituyen los componentes del modelo de negocios (segmento de clientes, propuesta de valor, canal, relación, flujo de ingresos, recursos clave, actividades clave, alianzas, estructura de costos) y que describe la hipótesis que se va a probar a través del proceso de desarrollo de cliente desde la parte del negocio e identificación de requerimientos y de desarrollo ágil desde el componente del producto tecnológico.

El proceso de desarrollo de cliente implica una continua dinámica de identificación y comunicación con el cliente, para identificar sus necesidades y revi- 
sar si efectivamente la idea de emprendimiento cumple con sus expectativas y es un producto susceptible de adquirirse. Está organizado en cuatro pasos que se muestran en la siguiente figura.

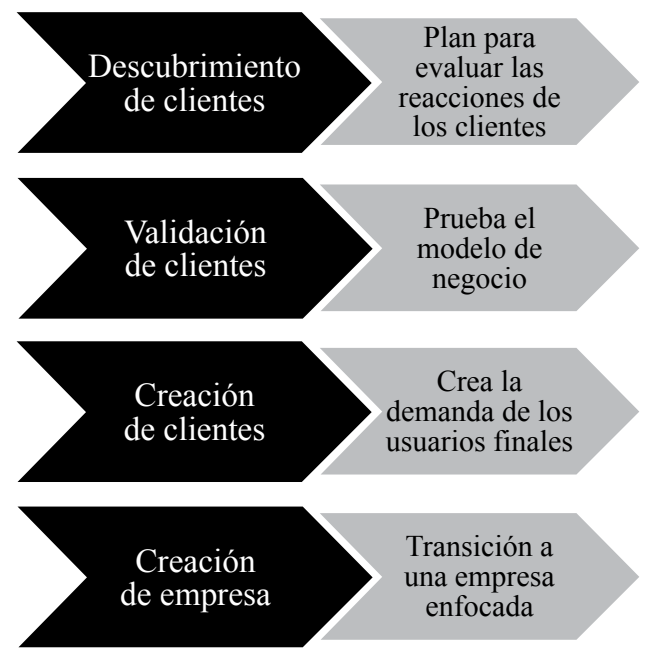

Figura 2. Proceso de desarrollo de cliente Fuente: los autores

En el proceso de desarrollo de cliente, la ejecución de entrevistas con los clientes y la sistematización de los resultados obtenidos a través de cambios realizados sobre el Business Model Canvas son la pieza clave de las ocho semanas de la fase "Ideación, prototipado y validación" del programa de emprendimiento Apps.co, al cual se incorporan personas con diferentes perfiles, conocimientos y perspectivas, que se agrupan libremente en equipos de dos a cuatro emprendedores.

La implementación del Lean LaunchPad del programa Apps.co que realiza SantanderApps se ejecuta a través de un proceso cuyo fin fundamental es proporcionar al equipo de emprendimiento los elementos necesarios para refinar su idea de negocio en coherencia con LLP, lo cual se muestra en la figura 3.

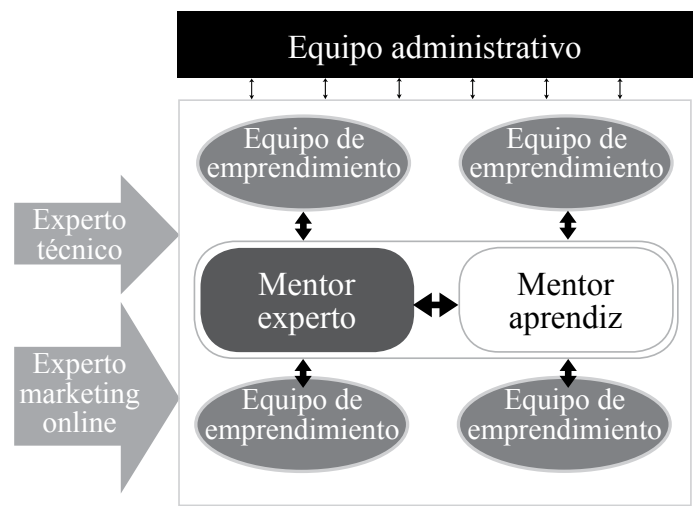

Figura 3. Proceso de emprendimiento en SantanderApps Fuente: los autores
El equipo de emprendimiento se encuentra en constante comunicación con el equipo de mentoría, el cual está conformado por un mentor experto y un mentor aprendiz. Si bien para Apps.co los emprendimientos son el indicador principal de su impacto, también es clave la formación de los mentores, para lograr que la transferencia de conocimiento se lleve a cabo de una manera eficaz y eficiente, por esta razón, en cada una de las iteraciones y para cada mentor experto se le define un acompañante en el proceso de mentoría, denominado mentor aprendiz. Dicho mentor tiene como función principal la asimilación de la metodología para su posterior divulgación.

Bajo las orientaciones del equipo de mentoría, el equipo de emprendimiento muestra los resultados de la validación de su modelo de negocio y toma las decisiones de pivote o modificación de su idea. Dentro de las estrategias utilizadas en el proceso de formación se encuentran: talleres de programación de apps, elevator pitch para comunicación de la idea, investigación de la competencia, búsqueda y entrevista de clientes, entre otras, que buscan acercar continuamente al equipo de emprendimiento con las características reales del mercado y de su competencia, además de foguearlo en la defensa de la misma, con base en el proceso de desarrollo de cliente.

Como asesores adicionales al proceso, se proporciona un experto técnico y un experto en marketing on-line. El primero colabora a los equipos de emprendimiento en la construcción de un mínimo producto viable (MPV) a través de la aplicación de metodologías de desarrollo ágil. Ese MPV es un medio de comunicación con el cliente que permite validar el alcance del producto software planteado. El segundo asesora a los equipos de emprendimiento para hacer un uso efectivo de los medios digitales para la promoción de su app en las diferentes tiendas on-line especializadas.

Adicionalmente, se cuenta con un equipo administrativo que facilita la logística necesaria para coordinar la ejecución de las actividades sin contratiempos, y verifica que se cumplan las condiciones establecidas por el Mintic para la ejecución del programa.

\section{Resultados}

A julio del 2013, la implementación realizada por SantanderApps - Unión Temporal UPB-UDI-CETICS - ha dado como resultado 40 iniciativas de emprendimiento que han superado la fase de "Ideación, prototipado y validación", y 21 iniciativas que están en proceso de 
formación. Además ha formado a 15 mentores en la metodología Lean LaunchPad.

Por otra parte, en la implementación realizada por SantanderApps - Unión Temporal UPB-UDI-CETICSse ha aplicado una metodología para identificar las lecciones aprendidas, que permiten hacer un análisis de los problemas, aciertos y aprendizajes que se generaron durante el proceso de entrenamiento, acompañamiento y asesoría de emprendedores.

A continuación se sintetizan los resultados obtenidos en los talleres de lecciones aprendidas en el que participaron mentores, emprendedores y comité técnico, ejercicio que ha contribuido al enriquecimiento y mejoramiento del proceso.

- La motivación es un aspecto fundamental para la continuidad de un emprendimiento. Cuando los integrantes de un equipo están motivados y poseen un objetivo claro, con mecanismos de comunicación directa, se logran más fácilmente las metas trazadas.

- El factor clave de éxito fue la comunicación permanente y el trabajo integrado de los mentores en proceso de formación, y posteriormente el canal de comunicación establecido con los emprendedores.

- Es importante disponer de un cronograma de actividades, que se debe ajustar e informar permanentemente a todos los implicados en los procesos de construcción del conocimiento.

- Con respecto a las ideas de emprendimiento:

- No asumir que se sabe qué quiere el cliente, es necesario preguntarle y validarlo.

- Se debe tener pasión por la idea, pero estar dispuesto a cambiarla según los resultados obtenidos en la investigación.

- Tomar decisiones basados en lo que tiene el mercado y analizar continuamente si el producto ofrecido satisface esas necesidades y si existen clientes dispuestos a pagar por él.

- Más importante que el desarrollo del aplicativo es realizar contactos y conocer su punto de vista acerca de la idea de negocio.

- Una síntesis del proceso formulada por los mismos emprendedores es:

- Identificar el mercado (potenciales clientes y competencia)

- Hacer contactos

- Sacar actas para la retroalimentación del cliente

- Según resultados, y si es necesario, se pivota la idea
- Armar la propuesta de valor de forma directa y concisa

\section{Discusión y conclusiones}

La experiencia de implementación del proceso de emprendimiento en el proyecto Apps.co gira en torno de la identificación y desarrollo de clientes para la idea de emprendimiento y la forma de monetización de la misma, lo que permite a los emprendedores centrar rápidamente su modelo de negocio y proporcionarles mayores posibilidades de éxito. Lo anterior es acorde con la implementación de la metodología Lean Launch Pad [6].

La apuesta nacional del Mintic por impulsar un eje de desarrollo económico centrado en el conocimiento, la tecnología y la creatividad es coherente con experiencias similares latinoamericanas (México, Chile) y con un área muy promisoria en el contexto internacional como son las apps para teléfonos inteligentes $[7,9,15]$.

La implementación realizada de la metodología LLP y las lecciones aprendidas hasta el momento abren la posibilidad para la incorporación al proceso de instrumentos que faciliten la identificación de características en los emprendedores, que faciliten el desarrollo de experiencias más exitosas, además de evaluar con mayor rigurosidad el impacto y viabilidad de cada una de las ideas de emprendimiento.

\section{Referencias}

[1] New Media Consortium, Informe Horizon, Nueva York: NMC, 2012.

[2] B. Lee, Mobile, Social, Big Data and Cloud, Gdansk, Poland: Gdansk University of Technology, Abr., 2012. [En línea]. Disponible en: http://www.slideshare.net/ infosharepl/burton-lee-stanford-university-mobilesocial-big-data-and-cloud-how-new-it-technologiesare-accelerating-innovation-and-scaling-startups-insilicon-valley-infoshare-2012

[3] Ministerio TIC de Colombia, Apps.co, Bogotá, Oct., 2012. [En línea]. Disponible en: http://apps.co/

[4] Santander Apps.co. "Apps.co-Ideación, prototipado y validación”, Nov., 2012. [En línea] Disponible en: http:// appssantander.co/portal 
[5] A. Hernández, En 2014 habrá 1700 millones de smartphones en el mundo, según un estudio, 2010. [En línea]. Disponible en http://www.tendencias21.net/ En-2014-habra-1-700-millones-de-smartphones-en-elmundo-segun-un-estudio_a4384.html

[6] S. Blank y B. Dorf, The Lean LaunchPad Online, Mar., 2012. Disponible en: http://steveblank.com/2012/09/06/ the-lean-launchpad-online/

[7] G. Anthes, "Invasion of the mobile Apps". Communication of the ACEM, Vol. 54, No. 9, 2011, pp. 16-18.

[8] M. Griggs, "Best Apps”. Discover, Vol. 33, No. 10, 2012, pp. 22-23.

[9] K. Lunau, "The touch-screnn school”. Macleans, 2013, pp. 59-62.

[10] J. Zelkowsky, “The TI-Nspire cas: A happy-medium mobile device for grades 8-16 mathematics classrooms" Techtrends, 2011, pp. 40-47.
[11] M. Gentile, "The Importance of Managing iPads in the Classroom”. Educaon Digest, 2012, pp. 11-13.

[12] J. Contreras, J. Herrera y S. Montoya, "Elementos instruccionales para el diseño y la producción de nateriales educativos móviles". Revista de Innovación Educativa, 2009, pp. 84-99.

[13] Law Practice, "13 Tech Tips for 2013". The Business of Practicing Law, 2013, pp. 28-37.

[14] C. Popesco y P. Simion, "Entrepreneurship Education and e-learning: A Perfect Match". Journal of Electrical \& Electronics Engineering, 2013, pp. 203-206.

[15] J. del Rey, “App time”. Inc., Dic., 2010-Ene., 2011, pp. 116-123.

[16] A. Osterwalder y I. Pigneur, "Business model generation”, EUA: John Wiley \& Sons, 2010. 